\title{
Differences in Binding and Monitoring Mechanisms Contribute to Lifespan Age Differences in False Memory
}

\author{
Yana Fandakova, Yee Lee Shing, and Ulman Lindenberger \\ Max Planck Institute for Human Development
}

\begin{abstract}
Based on a 2-component framework of episodic memory development across the lifespan (Shing \& Lindenberger, 2011), we examined the contribution of memory-related binding and monitoring processes to false memory susceptibility in childhood and old age. We administered a repeated continuous recognition task to children ( $N=20,10-12$ years), younger adults $(N=20,20-27$ years), and older adults $(N=21,68-76$ years). Participants saw the same set of unrelated word pairs in 3 consecutive runs and their task was to identify pair reoccurrences within runs. Across runs, correct detection of repeated pairs decreased in children only, whereas false recognition of lure pairs showed a greater increase in older adults than in children or younger adults. False recognition of rearranged pairs decreased across runs for all participants. This decrease was most pronounced in children, in particular for high-confidence memory errors. We conclude that memory binding mechanisms are sufficiently developed in children to facilitate memory monitoring and reduce false memory for associative information. In contrast, older adults show senescent impairments in both binding and monitoring mechanisms that both contribute to elevated illusory recollections in old age. We conclude that binding and monitoring processes during memory performance follow different developmental trajectories from childhood to old age.
\end{abstract}

Keywords: episodic memory, child development, aging, lifespan, false memory

False memory (i.e., erroneously remembering episodes that did not occur) is of great practical importance for eyewitness testimony and has received extensive attention both in the child developmental and aging literature (Ghetti \& Lee, 2011; Jacoby \& Rhodes, 2006). Age-related decreases in false memory are often observed in childhood samples; for instance, preschool children are more susceptible to false suggestions than older children (Ackil \& Zaragoza, 1995; Poole \& Lindsay, 1995). On the aging side, older adults are more susceptible to misinformation than younger

This article was published Online First December 31, 2012.

Yana Fandakova, Yee Lee Shing, and Ulman Lindenberger, Center for Lifespan Psychology, Max Planck Institute for Human Development, Berlin, Germany.

This study was carried out within the project "Cognitive and Neuronal Dynamics of Memory across the Lifespan (CONMEM)" at the Center for Lifespan Psychology, Max Planck Institute for Human Development, in partial fulfillment of the doctoral dissertation of the first author. During the work on her dissertation, Yana Fandakova was a predoctoral fellow of the International Max Planck Research School "The Life Course: Evolutionary and Ontogenetic Dynamics" (LIFE; www.imprs-life.mpg.de; participating institutions included MPI for Human Development, Humboldt-Universität zu Berlin, Freie Universität Berlin, University of Michigan, University of Virginia, University of Zurich). We thank Silvia Bunge, Pedro Paz-Alonso, Myriam Sander, and Markus Werkle-Bergner for valuable discussions.

Correspondence concerning this article should be addressed to Yee Lee Shing, Center for Lifespan Psychology, Max Planck Institute for Human Development, Lentzeallee 94, 14195 Berlin, Germany. E-mail: yshing@ mpib-berlin.mpg.de adults (Jacoby \& Rhodes, 2006; Roediger \& Geraci, 2007). ${ }^{1}$ Both children (6-9 years; Foley \& Johnson, 1985; Lindsay, Johnson, \& Kwon, 1991) and older adults (Hashtroudi, Johnson, \& Chrosniak, 1990; Henkel, Johnson, \& DeLeonardis, 1998) show difficulties when distinguishing events that actually happened in the past from those that were only imagined, probably reflecting children's and older adults' general difficulties in determining the source of different memory episodes (Mitchell \& Johnson, 2009; Spencer \& Raz, 1995). Furthermore, recent neuroimaging evidence suggests that age differences in the susceptibility to false memory, both in childhood (Paz-Alonso, Ghetti, Donohue, Goodman, \& Bunge, 2008) and old age (Dennis, Kim, \& Cabeza, 2007, 2008; Giovanello, Kensinger, Wong, \& Schacter, 2010), are related to differences in patterns of neuronal activation within the brain networks supporting the formation and retrieval of episodic memories (Simons \& Spiers, 2003), in particular the prefrontal (for 8-yearolds, 12-year-olds, and older adults) and medial temporal (for 8-year-olds and older adults) lobes.

So far only two studies have directly compared children and older adults on the propensity to commit memory errors (Rybash \& Hrubi-Bopp, 2000; Shing, Werkle-Bernger, Li, \& Lindenberger, 2009). Rybash and Hrubi-Bopp (2000) used a modified version of the Deese-Roediger-McDermott paradigm (DRM; Roediger \& McDermott, 1995) to investigate false memory deficits in firstgrade children, younger adults, and older adults. In the DRM

\footnotetext{
${ }^{1}$ In studies using the misinformation paradigm, after an initial encoding phase, participants are typically presented with some misleading information about details of the event (Loftus, Miller, \& Burns, 1978). Subsequently, participants are more likely to claim having seen the misinformation as a part of the initial learning phase (Lindsay \& Johnson, 1989; Loftus, 2005; Mitchell \& Zaragoza, 2001).
} 
paradigm, participants view a series of words that converge to a common nonpresented theme (e.g., bed, tired, pillow, rest). After studying these items, participants tend to falsely remember the nonpresented theme word (e.g., sleep) with high subjective confidence (Gallo, 2006). In this study, after studying DRM lists, participants were either asked to generate words related to the studied categories (generate condition) or to unrelated categories (control condition). The authors showed that children and older adults recalled more critical lures in the generate than the control condition, possibly reflecting difficulties in recollection-based processing (Ghetti \& Angelini, 2008; Healy, Light, \& Chung, 2005; see also Brainerd \& Reyna, 2004). Using an associative recognition paradigm with word pairs, Shing, Werkle-Bergner, Li, and Lindenberger $(2008,2009)$ found that older adults were disproportionately more likely to falsely endorse novel configurations of familiar words with high subjective confidence compared to children (10-12 years), teenagers (13-15 years), and younger adults. However, little is known about the commonalities and differences in the mechanisms underlying false memory between childhood and old age (cf. Craik \& Bialystock, 2006; Shing \& Lindenberger, 2011).

The findings reviewed above are largely in line with the twocomponent framework of episodic memory (Shing et al., 2008, 2010; Shing \& Lindenberger, 2011). According to this framework, episodic memory performance across the lifespan reflects the interplay between two independent but interacting associative and strategic components. The associative component depends on medial temporal functioning and refers to mechanisms that bind together different aspects of an event into a cohesive representation during the encoding, storage and retrieval of episodic memories. It is relatively mature by middle childhood (around age 6), as indicated by behavioral and neuroimaging evidence (Gogtay et al., 2004; Ofen et al., 2007; Sowell et al., 2003; but see Ghetti, DeMaster, et al., 2010; Gogtay et al., 2006), and undergoes senescent decline during adulthood and old age (Brehmer, Li, Muller, von Oertzen, \& Lindenberger, 2007; Cowan, Naveh-Benjamin, Kilb, \& Saults, 2006; Raz, Ghisletta, Rodrigue, Kennedy, \& Lindenberger, 2010; Raz et al., 2005; Sander, Werkle-Bergner, \& Lindenberger, 2011). The strategic component depends on prefrontal functioning and refers to control and organizational processes during encoding, as well as strategic search, monitoring and evaluation during retrieval. It shows prolonged development up to adolescence and young adulthood and also undergoes senescent decline in old age (Kausler, 1994; Ofen et al., 2007; Raz et al., 2005, 2010; Schneider \& Pressley, 1997).

The protracted development of the strategic component might contribute to children's increasing success in distinguishing the source of their memories or withstanding the effects of misinformation (Sluzenski, Newcombe, \& Ottinger, 2004). Strategic deficits might also be driving older adults increased propensity for false memory (Dodson \& Schacter, 2002). One of the processes supported by the strategic component that might be especially important for opposing false memories during retrieval is memory monitoring (Schacter, Norman, \& Koutstaal, 1998). Memory monitoring refers to the evaluation and control of information in episodic memory according to current task goals and decision criteria (Burgess \& Shallice, 1996; Mitchell \& Johnson, 2009; Moscovitch \& Winocur, 2002). Demand on monitoring processes is especially high whenever the presented information is associated with a high familiarity signal that needs to be overcome in order to reach a correct decision. Between preschool years and elementary school, the ability to withstand memory interference increases (Schneider \& Pressley, 1997). At the other end of the lifespan, older adults are more susceptible to proactive interference because of difficulties to avoid erroneous responses that are highly probable based on prior experience (Hay \& Jacoby, 1999; May, Hasher, Kane, 1999). However, so far no study has directly compared the ability to withstand interference in episodic memory between children and older adults, and its role in false memory across the lifespan.

Monitoring is also needed to subjectively assess the correctness of a potential memory response, for example in subjective confidence ratings, and serves as the basis of memory control (Koriat \& Goldsmith, 1996; Nelson \& Narens, 1990). Even though even younger children (e.g., 5-year-olds) can differentiate between correct and incorrect responses in their confidence judgments (Ghetti, 2003; Ghetti, Qin, \& Goodman, 2002), developmental increases in this ability are observed with increasing age (Ghetti, Lyons, Lazzarin, \& Cornoldi, 2008; Koriat, Goldsmith, Schneider, \& NakashDura, 2001; Roebers, 2002; Schneider \& Lockl, 2002). On the aging side, some evidence suggests similar miscalibration effects, such that older adults tend to be falsely confident about the accuracy of their recognition judgments (Dodson \& Krueger, 2006; Kelley \& Sahakyan, 2003). Older adults are especially prone to commit errors with high subjective confidence whenever recollection of specific details is necessary (Dodson, Bawa, \& Krueger, 2007). However, no such effects were observed in children (Shing et al., 2009), pointing to potential differences in episodic misrecollection between children and older adults.

Up to date, little is known about the effects of lifespan differences in the associative component on false memory and confidence calibration. As the associative and strategic components interact to support episodic memory, it is conceivable that more distinctive memory traces might facilitate monitoring processes and the rejection of highly familiar but irrelevant information. Given that the associative component is relatively mature by middle childhood, children's intact binding mechanisms may help to withstand interference and reduce their false memory levels for familiar associative information, leading to relatively low levels of high-confidence misrecollections (Shing et al., 2009). In contrast, older adults are assumed not to show such benefits, as their associative memory component is afflicted by senescent changes in the medial temporal lobes. We tested this lifespan dissociation directly by including children and older adults in the same experiment.

\section{The Present Study}

To address differences in memory-related binding and monitoring processes between children, younger adults, and older adults, we used a modified version of the repeated continuous recognition task (Schnider, von Daniken, \& Gutbrod, 1996). In each run, participants viewed the same task set of 84 word pairs (see Figure 1). A different subset of these word pairs was repeated in each run, and participants were asked to indicate exact pair reoccurrences within the ongoing run. Familiarity for the word pairs increased across runs, making it more difficult to discern whether each word pair was presented for the first time in the current run (termed lure 
Repeated Continuous Recognition Task

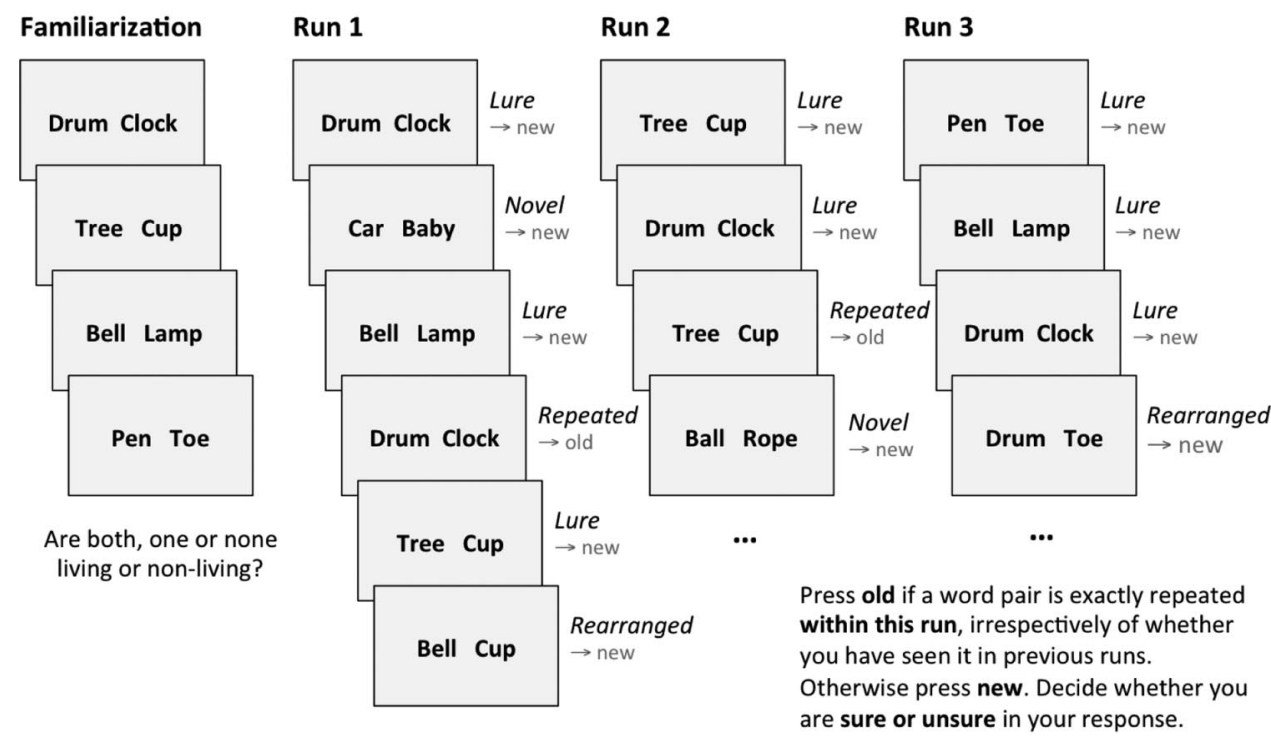

Figure 1. Experimental task design. Arrows at each trial indicate the expected correct response on the corresponding trial. Lure, novel, repeated and rearranged pairs were presented in each run of the task.

pairs) or was repeated in the current run (termed repeated pairs). We expected that across runs of the task participants would increasingly commit false alarms on the lure pairs. We hypothesized that this effect would be particularly pronounced in children and older adults, due to maturational and senescent changes in the strategic component, and in monitoring processes in particular.

Another subset of the word pairs were recombined within a run such that the two words of the pair were familiar but the conjunction between them was novel (rearranged pairs). While both children and older adults might have difficulties with memory monitoring and control, we hypothesized that children might be able to use their relatively mature associative component to reduce false responses for novel associative information. Hence, we expected that children and older adults will not differ in performance on rearranged pairs in the beginning of the task when demand on monitoring processes is maximized (Rotello \& Heit, 1999). We hypothesized that that children would commit fewer errors than older adults in later runs of the task because they would benefit more than older adults from multiple repetitions of the lure pairs across runs, presumably by strengthening memory representations for the lure pairs from which the single words are taken (cf. Kilb \& Naveh-Benjamin, 2011; Light, Patterson, Chung, \& Healy, 2004). Finally, we examined whether false memory differences between children and older adults are specifically related to committing high-confidence errors, and whether children's relatively developed associative component would facilitate their confidence calibration for novel conjunctions of familiar information.

\section{Method}

\section{Participants}

Participants in the present study were 20 children (aged 10-12 years), 20 younger adults (aged 20-27), and 21 older adults (aged
68-76). All participants were native German speakers. Children were attending the highest school track in Germany (the Gymnasium) that allows for university admission. Younger adults were university students, and older adults lived independently in the community and reported no major health issues. Older adults were screened for cognitive impairment with the Mini Mental State Examination (MMSE; $M=29.1, S D=0.89$, Range $=27-30$; Folstein, Folstein, $\&$ McHugh, 1975). Descriptive information of the participants is presented in Table 1. The participants were assessed on measures of perceptual speed (Digit Symbol; cf. Tewes, 1991; Wechsler, 1955) and crystallized intelligence (verbal knowledge; cf. Lehrl, 1977, 2005). Verbal knowledge increased continuously across the age groups, $F(2,57)=102.43, p<.05$. Age differences in perceptual speed followed an inverted U-shaped function, $F(2,57)=47.81, p<$ .05 , such that children and older adults did not differ from each other, and that both showed lower perceptual speed performance than younger adults. Hence, the three age groups showed age-typical performance on measures of perceptual speed and crystallized intelligence (Li et al., 2004).

\section{Materials}

A task set of 84 unrelated word pairs was formed from concrete German words. An additional set of 84 word pairs was used as

Table 1

Mean Descriptive Characteristics of Sample

\begin{tabular}{lcccccccc}
\hline & \multicolumn{2}{c}{ Children } & & \multicolumn{2}{c}{ Younger adults } & & \multicolumn{2}{c}{ Older adults } \\
\cline { 2 - 3 } \multicolumn{1}{c}{ Variable } & $M$ & $S D$ & & $M$ & $S D$ & & $M$ & $S D$ \\
\hline Age (years) & 10.78 & 0.4 & & 23.87 & 2.3 & & 72.22 & 2.6 \\
Male:female (\%) & $65: 35$ & & & $45: 55$ & & & $48: 52$ & \\
Digit symbol & 40.37 & 7.14 & & 68.10 & 12.37 & & 44.10 & 8.77 \\
Vocabulary & 13.00 & 3.77 & & 23.65 & 2.89 & & 28.57 & 3.75 \\
\hline
\end{tabular}


novel pairs in the main task. The word pairs were taken from a previous study on lifespan age differences in episodic memory (Shing et al., 2008) and five independent raters checked that the words constituting a pair were not phonetically, categorically or semantically related. The words were comparable on word frequency and length.

\section{Procedure}

At the beginning of the session participants were instructed and practiced the repeated continuous recognition task. Next, they completed the familiarization task, followed by three consecutive runs of the main task. Immediately before performing the modified continuous recognition task, each participant was familiarized with the task set of word pairs. During familiarization, each word pair was presented for $4 \mathrm{~s}$, followed by a fixation cross $(500 \mathrm{~ms})$. Participants had to indicate via button press whether none, one or both of the words depicted a living object.

Following this phase, participants performed three runs of the repeated continuous recognition task. A schematic of the task is depicted in Figure 1. In each run, the complete task set of word pairs was presented at least once (termed lure pairs), resulting in 84 lure pair trials. Due to the preceding familiarization task, the lure pairs were already familiar to the participants in run 1 . Additionally, 28 of the lure pairs were selected to reappear once in each run (termed repeated pairs). The participants' task was to respond "OLD" only to repeated pairs (i.e., those pairs that are exactly repeated within the same run) and "NEW" to all other pair types. For example, the word pair "Drum-Clock" (see Figure 1) is a lure pair when presented the first time in run 1 and is expected to receive a "NEW" response because this is the first occurrence of this word pair in this particular run. However, when "DrumClock" reappears in run 1 (see Figure 1) it is a repeated pair and is expected to receive an "OLD" response because it represents an exact repetition of a pair that has already been encountered in this particular run. Different subsets of word pairs from the task set were randomly selected to repeat in each run. For example, having been presented as a repeated pair in run 1, "Drum-Clock" would appear only as a lure pair in runs 2 and 3, respectively. In each run, another 28 of the lure pairs were selected to form recombined pairs, with the left word of a given lure pair presented in conjunction with the right word from another lure pair (termed rearranged pairs). The rearranged pairs were unique in the sense that a given recombination of words was never repeated within and across runs. For example, in run 1 both word pairs "Tree-Cup" and "BellLamp" are shown once as lure pairs (see Figure 1). The word pair "Bell-Cup" is shown later on and is a rearranged pair. Here, both the single words "Bell" and "Cup" are familiar to the participants because they have already presented in the run, but never in this conjunction. Hence, this word pair is expected to receive a "NEW" response. Finally, 28 word pairs that were completely novel to the participants (termed novel pairs) were presented in each run. For example, the word pair "Car-Baby" was presented only once in the whole experiment (see Figure 1) and was also not included in the familiarization phase.

Prior to the main task, participants were instructed that they would see word pairs and have to make "OLD" and "NEW" decisions on whether they have seen them before in the current run. Participants were instructed to indicate whether they had seen a word pair for the first or repeated time in the ongoing run, disregarding whether a pair appeared in any of the previous runs. They used four different buttons to give their response- "sure new," "unsure new," "unsure old," and "sure old." Presentation time for each word pair was fixed to $4 \mathrm{~s}$. Particularly for the second and third run, participants were reminded that they should base their decision only on the ongoing run, irrespective of whether the word pair has been presented before in the previous runs. In addition, the participants were informed that some of the words they have seen may reappear in a different configuration, in which case they were instructed to respond "NEW." An example was given for all conditions using word pairs that were not included in the actual task. For children and older adults, a real life example was given to make sure that they understood the novel configurations (e.g., buying different flavors of ice cream). Participants were given a practice task mimicking the real task. The setting of the practice task was identical to the main task with two exceptions. First, the word pairs used for practice were not included in the main task. Second, the runs were shorter and the task took about 5 min to complete. During the main modified continuous recognition task, there were brief breaks after the first and second runs (ca. 2-3 min), during which the participants were instructed for the upcoming run.

We hypothesized that lifespan differences in monitoring processes and representation quality would be differentially reflected in different types of word pairs. First, we expected that familiarity of the lure pairs would increase through their across-run repetitions, leading to increasing demands on memory monitoring to overcome the interference from multiple encounters of the same word pairs. For example, we expected that correctly rejecting the word pair "Drum-Clock" when presented for the first time in run 1 would require less memory monitoring than correctly rejecting it when occurring for the first time in Run 3, due to the increased interference from multiple presentations of this word pair in previous runs. In line with the prolonged development and early age-related decline of the strategic component (Shing et al., 2010), we predicted that children and older adults would have less efficient monitoring processes to overcome memory interference, resulting in a more pronounced increase in amount of false alarms to lure pairs from Run 1 to Run 3, compared to younger adults. Second, as memory for lure pairs is strengthened by across-run repetitions, we expected that the detection of novel word conjunctions - and hence the rejection of rearranged pairs-would become easier from run to run (Light et al., 2004). For instance, we expected that correctly rejecting the rearranged pair "Drum-Toe" in Run 3 would be easier than correctly rejecting the novel configuration "Bell-Cup" in Run 1 (see Figure 1). We hypothesized that this facilitation effect would be stronger in children, who can make use of a relatively functional associative component, compared to older adults, who show age-related decline in the ability to form and retrieve distinctive memory representations (Shing et al., 2010).

\section{Overview of Data Analysis}

Proportions of hits minus false alarms were computed for each participant and run (Snodgrass \& Corwin, 1988). In addition, we examined age differences in hits ("old" response to repeated pairs) 
and false alarms (FA; "old" response to lure, rearranged, or novel pairs) separately to better characterize memory changes at both ends of the lifespan. For analysis of confidence, proportions of FA with high-confidence ratings across all lure or rearranged pairs were computed. All measures of interest were analyzed using a repeated-measures analysis of variance (ANOVA) with age as a between-subjects factor (children vs. younger adults vs. older adults) and run as a within-subject factor. The Huynh-Feldt corrected degrees of freedom and significance values are reported when the sphericity assumption was not met (Huynh \& Feldt, 1976). An alpha level of .05 with Bonferroni correction for multiple comparisons was used for all statistical tests. Unless otherwise reported, post hoc comparisons were not significant. No outliers were found in any measure $\left(p<.001\right.$, two-tailed test) ${ }^{2}$

To ensure that the results reported below are not confounded by age differences in response bias, we examined response bias using the $\mathrm{Br}$ index (Snodgrass \& Corwin, 1988). In the repeated continuous recognition task lure and rearranged pairs are confounded with the main experimental manipulation. Therefore, we used novel pairs to compute the $\mathrm{Br}$ index as a measure of the tendency to respond "old" across age groups. Values of $\mathrm{Br}>0.5$ indicate a liberal bias (Snodgrass \& Corwin, 1988). An ANOVA revealed that the effects of run and the Run $\times$ Age interaction were not significant $(p>.5)$. The main effect of age group was reliable, $F(2,56)=4.72, p=.013$. Older adults $\left(M_{\mathrm{Br}}=.14, S D_{\mathrm{Br}}=.15\right)$ showed an overall more liberal bias than younger adults $\left(M_{\mathrm{Br}}=\right.$ $\left..04, S D_{\mathrm{Br}}=.08\right), t(29.14)=2.70, p=.012$. Response bias in children $\left(M_{\mathrm{Br}}=.07, S D_{\mathrm{Br}}=.08\right)$ did not differ from either older or younger adults $(p>.05)$. To control for possible effects of age differences in response bias, all results reported below were repeated using the $\mathrm{Br}$ index as a covariate in the ANOVAs. The main pattern of results did not change in these analyses.

\section{Results}

\section{Overall Memory Recognition Performance}

Separate ANOVAs examined recognition scores (Hits - FA) computed with FA for lure, rearranged, and novel pairs (see Table $2)$. For lure pairs, the main effect of run was significant, $F(1.8$, $106.2)=32.70, p=.001, \eta_{p}^{2}=.36$, indicating the reduction in performance across runs. This result is in line with the expected drop in performance for lure pairs with increasing interference from multiple repetitions of these word pairs across runs of the task. The main effect of age group was also significant, $F(2,58)=$ 11.89, $p=.001, \eta_{\mathrm{p}}^{2}=.29$. Children and older adults showed similar performance, and performed less well than younger adults, $t(38)=3.39, p=.001, d=1.07$ for children; $t(39)=4.55, p=$ $.001, d=1.4$ for older adults. The Age Group $\times$ Run interaction was not reliable, $F(3.7,106.2)=0.95, p=.44, \eta_{p}^{2}=.03$.

For recognition scores with rearranged pairs, performance of all age groups increased across runs, $F(1.9,111.4)=17.94, p=.001$, $\eta_{\mathrm{p}}^{2}=.24$, in line with the expected facilitation of rejecting these pairs with more distinctive representations of the pairs from which the single words are taken. The main effect of age group was also reliable, $F(2,58)=27.57, p=.001, \eta_{\mathrm{p}}^{2}=.49$, but not the Age Group $\times$ Run interaction, $F(3.8,111.4)=0.15, p=.96, \eta_{\mathrm{p}}^{2}=.01$ (see Table 2). Children and older adults showed similar performance on rearranged pairs, that was significantly lower than
Table 2

Mean Hit-False Alarm (FA) With Lure, Rearranged, and Novel Pairs Across Runs of the Modified Continuous Recognition Task Across Age Groups

\begin{tabular}{|c|c|c|c|c|c|c|}
\hline \multirow[b]{2}{*}{ Variable } & \multicolumn{2}{|c|}{ Children } & \multicolumn{2}{|c|}{$\begin{array}{l}\text { Younger } \\
\text { adults }\end{array}$} & \multicolumn{2}{|c|}{ Older adults } \\
\hline & $M$ & $S D$ & $M$ & $S D$ & $M$ & $S D$ \\
\hline \multicolumn{7}{|l|}{ Hit-FA Lure } \\
\hline Run 1 & .58 & .14 & .70 & .16 & .57 & .11 \\
\hline Run 2 & .49 & .12 & .67 & .16 & .48 & .10 \\
\hline Run 3 & .44 & .15 & .56 & .19 & .39 & .13 \\
\hline \multicolumn{7}{|c|}{ Hit-FA Rearranged } \\
\hline Run 1 & .30 & .17 & .59 & .23 & .18 & .09 \\
\hline Run 2 & .35 & .21 & .67 & .27 & .24 & .19 \\
\hline Run 3 & .44 & .20 & .72 & .26 & .30 & .22 \\
\hline \multicolumn{7}{|l|}{ Hit-FA Novel } \\
\hline Run 1 & .72 & .15 & .82 & .14 & .78 & .10 \\
\hline Run 2 & .61 & .17 & .84 & .14 & .75 & .10 \\
\hline Run 3 & .60 & .21 & .82 & .13 & .75 & .12 \\
\hline \multicolumn{7}{|l|}{ Novel FA } \\
\hline Run 1 & .02 & .03 & .01 & .02 & .04 & .05 \\
\hline Run 2 & .03 & .05 & .01 & .03 & .03 & .04 \\
\hline Run 3 & .04 & .09 & .003 & .01 & .03 & .03 \\
\hline
\end{tabular}

younger adults' performance, $t(38)=4.55, p=.001, d=1.44$ for children; $t(29.8)=6.95, p=.001, d=2.18$ for older adults.

For recognition scores with novel pairs, performance of all age groups increased across runs, $F(2,116)=5.71, p=.004, \eta_{\mathrm{p}}^{2}=$ .09 . The main effect of age group was reliable, $F(2,58)=27.57$, $p=.001, \eta_{\mathrm{p}}^{2}=.49$, as well as the Age $\times$ Run interaction, $F(4$, 116) $=3.96, p=.005, \eta_{\mathrm{p}}^{2}=.12$ (see Table 2). While the age groups did not differ in Run 1, in Run 2 children showed lower recognition than younger adults, $t(38)=4.50, p=.001, d=1.42$, and older adults, $t(29.8)=3.06, p=.010, d=0.96$. In Run 3, children's performance was again lower than both younger adults, $t(31.63)=3.96, p=.001, d=1.25$, and older adults, $t(29.7)=$ $2.79, p=.011, d=0.88$.

To summarize, when computed with lure and rearranged pairs, overall recognition scores were lower in children and older adults than in younger adults, with no reliable differences between children and older adults. In contrast, when computed with novel pairs, children's recognition performance was lower than younger and older adults. Given our expectations regarding lifespan differences in errors patterns, hits and false alarms were examined separately in the next set of analyses.

\section{Veridical and False Memory}

We expected that lifespan differences in monitoring processes and quality of memory representations would be differentially reflected in performance to lure and rearranged pairs. Specifically, we expected an increase in FA rates to lure pairs with increasing interference from previous presentations across runs. In contrast, we hypothesized that FA rates to rearranged pairs would decrease across runs, reflecting strengthening of the memory

\footnotetext{
${ }^{2}$ In all age groups, reaction time was below the maximal word pair presentation time of $4 \mathrm{~s}$. This was true for all conditions and runs of the present experiment.
} 
representations for lure pairs through their repeated presentation. To directly assess these predictions, we performed separate ANOVAs on hits and FA rates. The results of these analyses are presented in Figure 2.

Hits (Figure 2A). The main effects of run, $F(1.9,109.1)=$ $7.45, p=.002, \eta_{\mathrm{p}}^{2}=.11$, and age group, $F(2,58)=8.94, p=$ $.001, \eta_{\mathrm{p}}^{2}=.24$, were significant. Moreover, the Run $\times$ Age Group interaction was reliable, $F(3.8,109.1)=4.89, p=.002, \eta_{\mathrm{p}}^{2}=.14$. While the age groups did not differ in Run 1, in Run 2 children showed lower hit rate than younger adults, $t(38)=4.39, p=.001$, $d=1.38$, and older adults, $t(30.3)=3.39, p=.003, d=1.08$. In Run 3, children also made less hits than both younger adults, $t(38)=3.64, p=.001, d=1.11$, and older adults, $t(32.01)=$ $2.88, p=.009, d=0.89$. Taken together, while younger and older adults did not differ in their ability to detect repeated word pairs within a run, children showed lower performance on repeated pairs in Runs 2 and 3.

Lure FA (Figure 2B). There were significant main effects of run, $F(1.8,100.9)=19.10, p=001, \eta_{\mathrm{p}}^{2}=.25$, and age group, $F(2$, $58)=8.85, p=.001, \eta_{\mathrm{p}}^{2}=.23$. The Age Group $\times$ Run interaction was also reliable, $F(3.5,100.9)=3.21, p=.023, \eta_{\mathrm{p}}^{2}=.10$. Post hoc tests revealed that in Run 1 older adults committed more FA than younger adults, $t(39)=3.36, p=.005, d=1.07$. However, in Run 2 older adults made more FA than both children, $t(39)=$ $4.08, p=.001, d=1.24$, and younger adults, $t(39)=3.24, p=$ $.003, d=1.00$. In Run 3 older adults again showed higher FA rate than children, $t(39)=3.76, p=.002, d=1.15$, and younger adults, $t(39)=2.40, p=.049, d=0.75$. Taken together, with increasing interference from multiple repetitions of the lure pairs across runs, participants became more likely to erroneously endorse a lure pair as already seen in the ongoing run. Of note, this tendency was stronger in older adults compared to both children and younger adults.

Rearranged FA (Figure 2C). There was a reliable effect of run, $F(1.8,107)=41.05, p=.001, \eta_{\mathrm{p}}^{2}=.41$, indicating an overall decrease in FA to rearranged pairs across runs. The effect of age group was also significant, $F(2,58)=31.06, p=.001, \eta_{\mathrm{p}}^{2}=.52$. Younger adults demonstrated a lower FA rate than both children, $t(38)=3.02, p=.005, d=0.96$, and older adults, $t(39)=7.94$, $p=.001, d=2.48$. In addition, children committed less rearranged FA than older adults, $t(39)=4.66, p=.012, d=1.45$. The Age $\times$ Run interaction showed a trend toward statistical significance, $F(3.7,107)=2.25, p=.073, \eta_{\mathrm{p}}^{2}=.07$, reflecting the tendency in the three age groups to show distinctive pattern of change in memory performance across runs. In particular, a closer examination of Figure 2C suggests that children, but not older adults, showed a more pronounced decrease in the amount of errors to rearranged pairs from Run 1 to Run 2.

Novel FA (Table 2). For FA to novel pairs, only the main effect of age group was reliable, $F(2,56)=3.49, p=.037, \eta_{\mathrm{p}}^{2}=$ .11. Older adults committed reliably more novel FA than younger adults, $t(26.93)=3.07, p=.045, d=1.07$. Children did not differ reliably from both younger and older adults. There were no reliable effects of run, $F(2,112)=0.17, p=.84, \eta_{\mathrm{p}}^{2}=.003$ or an Age Group $\times$ Run interaction, $F(4,112)=0.19, p=.94, \eta_{\mathrm{p}}^{2}=.007$.

To examine to what extent age differences in performance on lure and rearranged pairs was affected by age differences in false alarm rates to novel pairs, the hit rate for repeated pairs and the false alarm rates for rearranged and for lure pairs were corrected
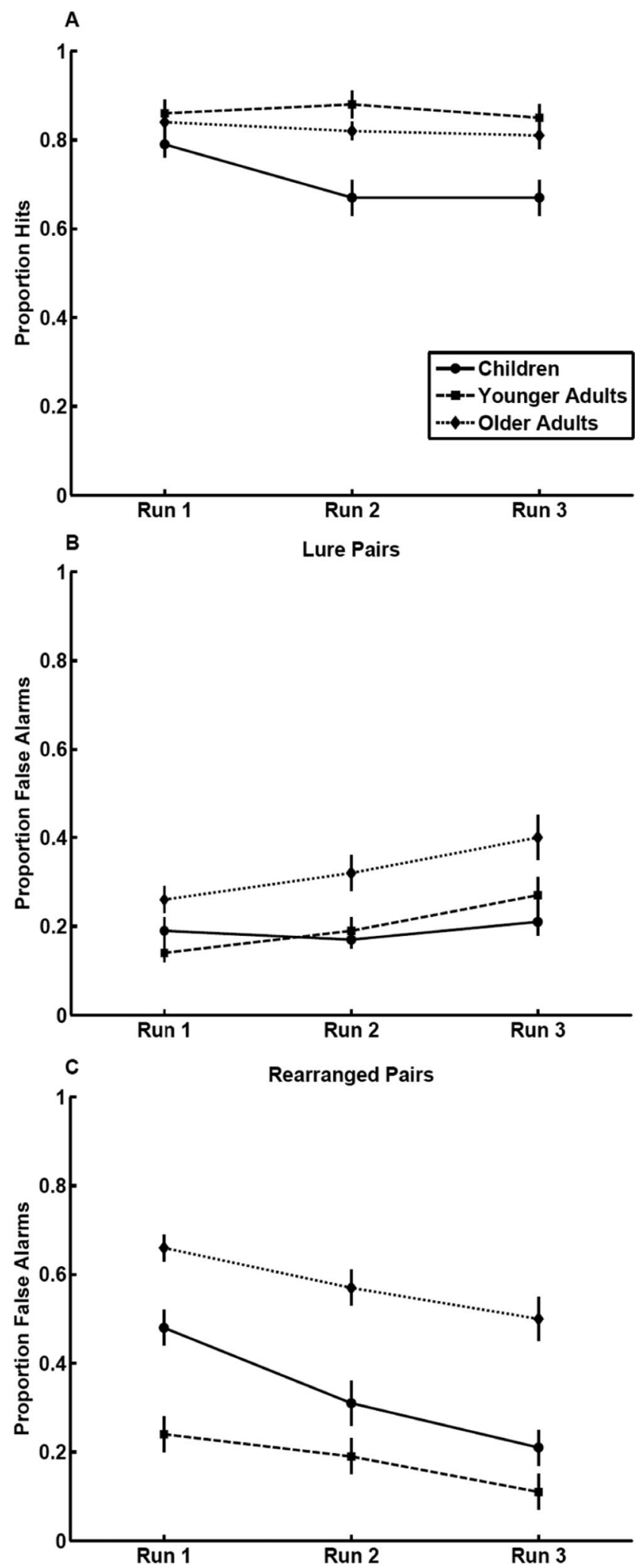

Figure 2. Mean proportion of hits to repeated pairs (A), false alarms to lure pairs (B), and false alarms to rearranged pairs (C) across runs of the modified continuous recognition task. Error bars represent standard error of the mean. 
by subtracting from them the false alarms for novel pairs. All analyses were repeated with the corrected measures (see Light et al., 2004), and the results were identical to the results reported here.

\section{High-Confidence Memory Errors}

To determine the degree to which age differences in FA to lure and rearranged pairs were driven by committing errors with high subjective confidence, we compared "sure old" responses for repeated, lure, and rearranged pairs in separate ANOVAs (see Figure 3).

High-confidence hits (Figure 3A). The main effects of run and age group showed a trend toward statistical significance, $F(1.9,110)=2.88, p=.063, \eta_{\mathrm{p}}^{2}=.05$ and $F(2,58)=2.47, p=$ $.093, \eta_{\mathrm{p}}^{2}=.08$, respectively. The Age Group $\times$ Run interaction was statistically reliable, $F(3.8,110)=6.82, p=.001, \eta_{\mathrm{p}}^{2}=.19$. Post hoc comparisons revealed that children's rate of highconfidence hits was significantly lower than younger adults in Run $2, t(38)=2.88, p=.008, d=0.90$. There were no differences between younger and older adults. Importantly, no differences between children and older adults were found in any of the runs, indicating that subsequent analysis of high-confidence lure and rearranged FA are not driven by differences in the general tendency to report high confidence.

High-confidence lure FA (Figure 3B). An ANOVA revealed a main effects of run, $F(1.7,99.9)=24.09, p=.001, \eta_{\mathrm{p}}^{2}=.29$ and age group, $F(2,58)=4.72, p=.013, \eta_{\mathrm{p}}^{2}=.14$. The Age Group $\times$ Run interaction was also reliable, $F(3.4,99.9)=5.16, p=.001$, $\eta_{\mathrm{p}}^{2}=.15$. While in Run 1 the three age groups did not differ in the amount of sure FA, in Run 2 older adults committed more highconfidence responses than children, $t(39)=2.40, p=.035, d=$ 0.69 , and younger adults, $t(39)=2.72, p=.023, d=0.86$. Similarly, in Run 3 older adults showed higher sure FA rate than children, $t(37.1)=2.55, p=.018, d=0.78$, and younger adults, $t(30.3)=3.16, p=.007, d=0.94$. In sum, the three age groups showed similar amounts of FA to lure pairs in Run 1. However, only older adults were increasingly more likely to erroneously endorse these pairs with high subjective confidence in later runs.

High-confidence rearranged FA (Figure 3C). The main effects of run and age group were statistically reliable, $F(1.7$, $96.6)=11.60, p=.001, \eta_{\mathrm{p}}^{2}=.17$ and $F(2,58)=10.29, p=.001$, $\eta_{\mathrm{p}}^{2}=.26$, respectively. Moreover, the Age Group $\times$ Run interaction was significant, $F(3.3,96.6)=2.59, p=.050, \eta_{\mathrm{p}}^{2}=.08$, reflecting that there were pronounced differences among the age groups in illusory memories committed with high subjective confidence across runs of the task. Of note, in Run 1 children and older adults did not differ from each other and both committed more sure FA than younger adults, $t(29.3)=3.21, p=.015, d=$ 1.01 for children; $t(30.78)=3.81, p=.003, d=1.18$ for older adults. However, in Run 2 this pattern changed, and older adults committed more sure FA compared to both younger adults, $t(31)=$ 4.43, $p=.001, d=1.36$, and children, $t(39)=2.34, p=.032, d=$ 0.73 , who did not differ from each other. In Run 3 , again, older adults made more sure FA than both children, $t(28.1)=2.54, p=$ $.017, d=0.78$, and younger adults, $t(26.2)=3.93, p=.001, d=$ 1.19. Taken together, the pattern of high-confidence FA to rearranged pairs differed between children and older adults across runs of the task. While both groups started off with a similar FA rate,
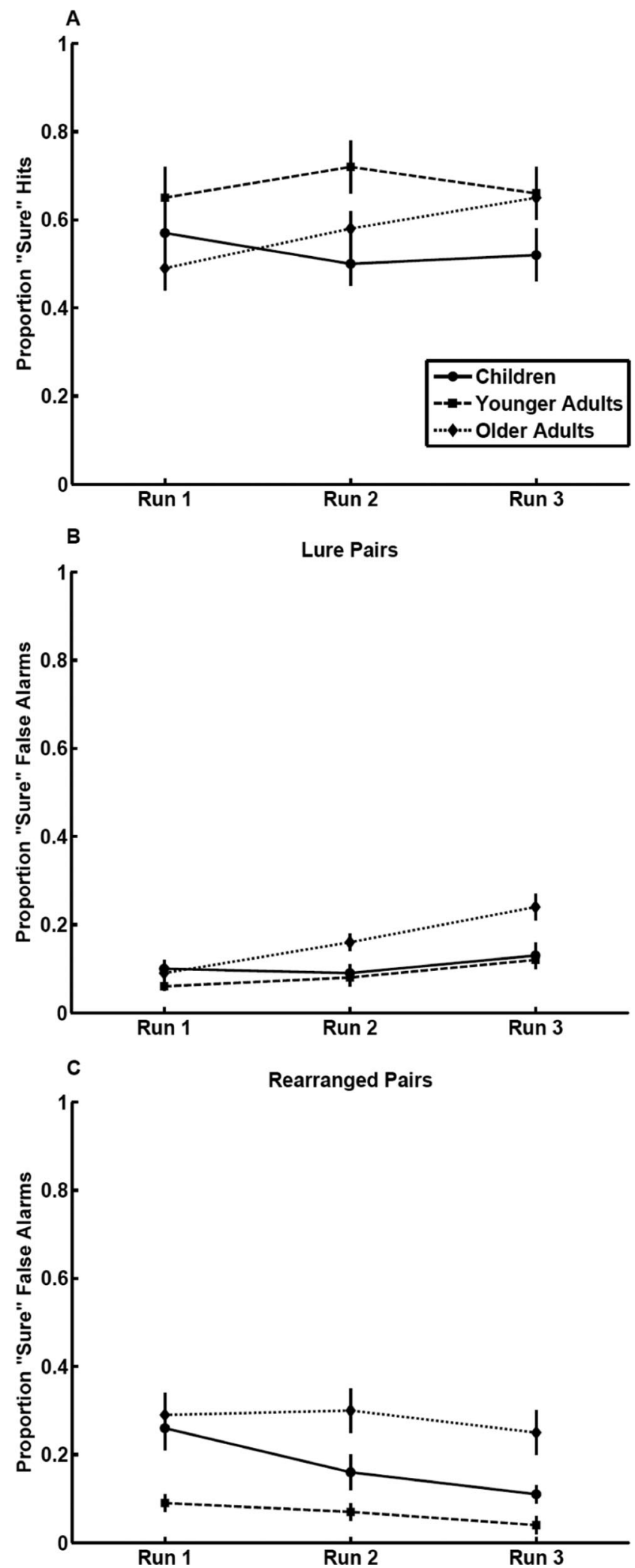

Figure 3. Mean proportion of high-confidence hits to repeated pairs (A), high-confidence false alarms to lure pairs (B), and high-confidence false alarms to rearranged pairs (C) across runs of the modified continuous recognition task. Error bars represent standard error of the mean. 
already in Run 2 children decreased their high-confidence errors to the level of younger adults. In contrast, older adults did not show such a decrease in high-confidence FA and were more likely to commit high-confidence errors to novel configurations of familiar words than the remaining age groups throughout all task runs.

\section{Discussion}

We examined the contribution of age differences in binding and monitoring processes to false memory for currently relevant associative information across the lifespan. Overall, children and older adults showed lower recognition performance than younger adults, but differed in their patterns of hits and false alarms across runs of the task. Correct detection of repeated pairs decreased in children only. In contrast, false recognition of lure pairs was higher in older adults than in children or younger adults. False recognition of rearranged pairs decreased across runs for all participants. This decrease was most pronounced in children, in particular regarding memory errors committed with high subjective confidence. Taken together, our findings indicate that binding and monitoring processes followed different developmental trajectories from childhood to old age.

We assumed that as lure pairs are presented in each run, correctly rejecting these pairs in later runs would require greater memory monitoring to overcome interference from multiple presentations in previous runs. We expected that children and older adults would show greater difficulties in correctly rejecting lure pairs as presented for the first time in the current run, reflecting maturational and senescent changes in memory monitoring. As expected, older adults committed more FA to lure pairs than children and younger adults. In contrast, children showed lower hit rates than the adult participants (Shing et al., 2008). These findings provide direct evidence for a lifespan dissociation of the mechanisms that characterize memory performance in childhood and old age. Some of these mechanisms may involve age differences in the enhancement of relevant and suppression of irrelevant information, respectively. A series of experiments by Gazzaley, Cooney, Rissman, and D'Esposito (2005) showed that old age is related to declines in suppression of irrelevant information, while enhancement of relevant information is generally preserved (see also Hasher \& Zacks, 1988). Conversely, Wendelken, Baym, Gazzaley, and Bunge (2011) found that top-down enhancement of relevant information continues to increase in the course of middle childhood. The present findings confirm and extend these earlier results by directly comparing children and older adults within the same study. According to this interpretation, children may have difficulties enhancing signals from episodic memory, resulting in lower detection of repeated pairs under conditions of high interference. In contrast, older adults may have difficulties inhibiting currently irrelevant information, hence committing more memory errors. An important route for future research is to find out whether this lifespan dissociation in behavioral performance can be mapped onto distinctive control processes during memory retrieval that are supported by different PFC subregions (Dobbins, Foley, Schacter, \& Wagner, 2002) and might exhibit distinct trajectories of functional recruitment across the lifespan (Sowell et al., 2004).

For rearranged pairs, we hypothesized that children would benefit more than older adults from repeated presentations of the original lure pairs across runs, due to their relatively functional associative component. Compared to older adults, children's greater ability to benefit from repeated exposure was expected to result in a greater reduction in FA for rearranged pairs (Light et al., 2004). The data were fully consistent with this prediction. From the first to the second runs of the task, the reduction in rearranged FA was almost twice as large for children than for older adults (see Figure $2 \mathrm{C}$ ). The difference between the FA rates of younger and older adults depicted in Figure $2 \mathrm{C}$ is particularly striking. For older adults, repeated presentation of the lure pairs had no beneficial effects for detecting novel configurations (Light et al., 2004). This result corroborates on previous findings of increased false memory in old age (cf. Bender, Naveh-Benjamin, \& Raz, 2010; Castel \& Craik, 2003). It underscores the importance of identifying the neural mechanisms underlying age-related deficits in evaluating novel configurations of familiar information. While older adults may have deficits in the basic novelty detection mechanisms in the hippocampus and the surrounding cortex (Kumaran \& Maguire, 2009), deficits in control processes confined to prefrontal regions may also be involved.

The performance difference between children and older adults was especially marked with regard to high-confidence FA (see Figure 3C). Here, in Run 1, both children and older adults were more likely than younger adults to commit high-confidence FA, corroborating earlier findings of protracted maturation of memory monitoring abilities in childhood (Ghetti, Castelli, \& Lyons, 2010; Koriat \& Ackerman, 2010; Schneider, 1998), and of senescent decline in these abilities in old age (Chua, Schacter, \& Sperling, 2009; Dodson et al., 2007). Of note, while older adults showed little or no change in high-confidence errors to rearranged pairs across runs, children decreased their highconfidence errors and did not differ from younger adults in the second and third runs of the task (see Figure 3C). These results suggest that when given the possibility to repeatedly encode the pairs across runs, children were able to make use of a relatively well functioning binding (Shing et al., 2008; Sluzenski, Newcombe, \& Kovacs, 2006) to build distinctive representations of which two words belong to a lure pair and use them to correctly reject new rearrangements and calibrate subjective confidence. Crucially, as predicted by the twocomponent framework, no such changes were observed for older adults. To our knowledge, this is the first study to document that false memory in childhood can be reduced considerably through support of basic binding processes. These results should be interpreted with caution as first initial evidence and remain to be replicated. However, given recent evidence that structural and functional development of the hippocampal subregions is not homogeneous and may be related to age-graded changes in memory ability during middle childhood (Ghetti, DeMaster, Yonelinas, \& Bunge, 2010; Gogtay et al., 2006), it seems worthwhile to further examine when and how children start to make use of their binding potential to facilitate strategic processes.

Finally, a possible caveat of the current study must be taken into consideration. We assumed that the increase in memory errors for lure pairs across runs reflects nonoptimal memory monitoring processes as opposed to decrease in errors for rearranged pairs across runs that presumably reflects the ability to form and retrieve distinctive memory representations. However, it should be taken into consideration that the mapping of associative and strategic mechanisms on different types of word pairs is not fully orthogonal. Rather, performance on both lure and rearranged pairs depends on both the ability to bind the different aspects of an episode to a coherent representation, as well as on control processes operating on these representations (Shing et al., 2010; Mitchell \& Johnson, 2009; Simons \& Spiers, 2003). Therefore, 
our assumptions should be understood in relative terms. Given the high similarity among runs, we assume that attribution of a lure pair to the specific context (i.e., run) in which it was previously presented depends on controlled strategic processing, as suggested by the source monitoring framework (Johnson, Hashtroudi, \& Lindsay, 1993; Mitchell \& Johnson, 2009). However, we cannot rule out that older adults, due to aging-induced deficits in pair-to-run binding, may rely more on familiarity-based processing leading to greater demands on monitoring mechanisms, which also decline in old age. With the current paradigm we cannot fully disentangle these various sources of deficits for lure pair processing in old age. This question should be addressed in future research as it is also highly relevant for understanding age-related deficits in memory for temporal context, which has so far received only limited attention in the aging literature (but see Naveh-Benjamin, 1990; Fabiani \& Friedman, 1997; Kausler, Salthouse, \& Saults, 1988; Rajah \& McIntosh, 2008).

Similarly, it has been shown that repeated presentation facilitates performance on rearranged pairs (e.g., Light et al., 2004), suggesting that improvement in performance for rearranged pairs is relatively more dependent on quality of memory representations. This does not exclude the possibility that more distinctive memory representations facilitate performance by decreasing the demand on recall-to-reject monitoring processes. We cannot tease these two influences apart in the context of the current paradigm. Future studies should investigate the cognitive and neural mechanisms by which strategic and associative components interact to facilitate performance. In particular, the improvement in confidence calibration that we observed for children in the present study may reflect these interactions.

To conclude, the ability to withstand interference in episodic memory shows protracted maturation in childhood and senescent decline in late adulthood. However, children and older adults suffer from interference in markedly different ways. Future studies need to delineate age differences in the relative contributions of enhancement and inhibition to false memory. Of particular interest is research on the interplay between medial temporal and prefrontal development in middle childhood, as it may help to understand how evolving binding mechanisms and strategic monitoring processes mutually influence each other in the control of memory contents.

\section{References}

Ackil, J. K., \& Zaragoza, M. S. (1995). Developmental differences in eyewitness suggestibility and memory for source. Journal of Experimental Child Psychology, 60, 57-83. doi:10.1006/jecp.1995.1031

Bender, A. R., Naveh-Benjamin, M., \& Raz, N. (2010). Associative deficit in recognition memory in a lifespan sample of healthy adults. Psychology and Aging, 25, 940-948. doi:10.1037/a0020595

Brainerd, C. J., \& Reyna, V. F. (2004). Fuzzy-trace theory and memory development. Developmental Review, 24, 396-439. doi:10.1016/j.dr 2004.08 .005

Brehmer, Y., Li, S. C., Muller, V., von Oertzen, T., \& Lindenberger, U. (2007). Memory plasticity across the lifespan: Uncovering children's latent potential. Developmental Psychology, 43, 465-478. doi:10.1037/ 0012-1649.43.2.465

Burgess, P. W., \& Shallice, T. (1996). Confabulation and the control of recollection. Memory, 4, 359-412. doi:10.1080/096582196388906

Castel, A. D., \& Craik, F. I. (2003). The effects of aging and divided attention on memory for item and associative information. Psychology and Aging, 18, 873-885. doi:10.1037/0882-7974.18.4.873
Chua, E. F., Schacter, D. L., \& Sperling, R. A. (2009). Neural basis for recognition confidence in younger and older adults. Psychology and Aging, 24, 139-153. doi:10.1037/a0014029

Cowan, N., Naveh-Benjamin, M., Kilb, A., \& Saults, J. S. (2006). Lifespan development of visual working memory: When is feature binding difficult? Developmental Psychology, 42, 1089-1102. doi:10.1037/ 0012-1649.42.6.1089

Craik, F. I. M., \& Bialystok, E. (2006). Cognition through the lifespan: Mechanisms of change. Trends in Cognitive Sciences, 10, 131-138. doi:10.1016/j.tics.2006.01.007

Dennis, N. A., Kim, H., \& Cabeza, R. (2007). Effects of aging on true and false memory formation: An fMRI study. Neuropsychologia, 45, $3157-$ 3166. doi:10.1016/j.neuropsychologia.2007.07.003

Dennis, N. A., Kim, H., \& Cabeza, R. (2008). Age-related differences in brain activity during true and false memory retrieval. Journal of Cognitive Neuroscience, 20, 1390-1402. doi:10.1162/jocn.2008.20096

Dobbins, I. G., Foley, H., Schacter, D. L., \& Wagner, A. D. (2002). Executive control during episodic retrieval: Multiple prefrontal processes subserve source memory. Neuron, 35, 989-996. doi: S0896627302008589

Dodson, C. S., Bawa, S., \& Krueger, L. E. (2007). Aging, metamemory, and high-confidence errors: A misrecollection account. Psychology and Aging, 22, 122-133. doi:10.1037/0882-7974.22.1.122

Dodson, C. S., \& Krueger, L. E. (2006). I misremember it well: Why older adults are unreliable eyewitnesses. Psychonomic Bulletin \& Review, 13, 770-775. doi:10.3758/BF03193995

Dodson, C. S., \& Schacter, D. L. (2002). Aging and strategic retrieval processes: Reducing false memories with a distinctiveness heuristic Psychology and Aging, 17, 405-415. doi:10.1037/0882-7974.17.3.405

Fabiani, M., \& Friedman, D. (1997). Dissociations between memory for temporal order and recognition memory in aging. Neuropsychologie, 35 , 129-141.

Foley, M. A., \& Johnson, M. K. (1985). Confusions between memories for performed and imagined actions: A developmental comparison. Child Development, 56, 1145-1155. doi:10.2307/1130229

Folstein, M. F., Folstein, S. F., \& McHugh, P. R. (1975). "Mini-mental state": A practical method for grading the cognitive state of patients for the clinician. Journal of Psychiatric Research, 12, 189-198. doi: 10.1016/0022-3956(75)90026-6

Gallo, D. A. (2006). Associative illusions of memory: False memory research in DRM and related tasks. New York, NY: Psychology Press.

Gazzaley, A., Cooney, J. W., Rissman, J., \& D'Esposito, M. (2005). Top-down suppression deficit underlies working memory impairment in normal aging. Nature Neuroscience, 8, 1298-1300. doi:10.1038/nn1543

Ghetti, S. (2003). Memory for nonoccurrences: The role of metacognition. Journal of Memory and Language, 48, 722-739.

Ghetti, S., \& Angelini, L. (2008). The development of recollection and familiarity in childhood and adolescence: Evidence from the dualprocess signal detection model. Child Development, 79, 339-358. doi: 10.1111/j.1467-8624.2007.01129.x

Ghetti, S., Castelli, P., \& Lyons, K. E. (2010). Knowing about not remembering: Developmental dissociations in lack-of-memory monitoring. Developmental Science, 13, 611-621. doi:10.1111/j.1467-7687.2009 .00908.x

Ghetti, S., DeMaster, D. M., Yonelinas, A. P., \& Bunge, S. A. (2010) Developmental differences in medial temporal lobe function during memory encoding. The Journal of Neuroscience, 30, 9548-9556. doi: 10.1523/JNEUROSCI.3500-09.2010

Ghetti, S., \& Lee, J. (2011). Children's episodic memory. Wiley Interdisciplinary Reviews: Cognitive Science, 2, 365-373. doi:10.1002/wcs.114

Ghetti, S., Lyons, K. E., Lazzarin, F., \& Cornoldi, C. (2008). The development of metamemory monitoring during retrieval: The case of memory strength and memory absence. Journal of Experimental Child Psychology, 99, 157-181. doi:10.1016/j.jecp.2007.11.001 
Ghetti, S., Qin, J., \& Goodman, G. S. (2002). False memories in children and adults: Age, distinctiveness, and subjective experience. Developmental Psychology, 38, 705-718.

Giovanello, K. S., Kensinger, E. A., Wong, A. T., \& Schacter, D. L. (2010). Age-related neural changes during memory conjunction errors. Journal of Cognitive Neuroscience, 22, 1348-1361. doi:10.1162/jocn .2009 .21274

Gogtay, N., Giedd, J. N., Lusk, L., Hayashi, K. M., Greenstein, D., Vaituzis, A. C., . . Thompson, P. M. (2004). Dynamic mapping of human cortical development during childhood through early adulthood. PNAS Proceedings of the National Academy of Sciences of the United States of America, 101, 8174-8179. doi:10.1073/pnas.0402680101

Gogtay, N., Nugent, T. F., 3rd, Herman, D. H., Ordonez, A., Greenstein, D., Hayashi, K. M., . . . Thompson, P. M. (2006). Dynamic mapping of normal human hippocampal development. Hippocampus, 16, 664-672. doi:10.1002/hipo.20193

Hasher, L., \& Zacks, R. T. (1988). Working memory, comprehension, and aging: A review and a new view. In G. H. Bower (Ed.), The psychology of learning and motivation (Vol. 22, pp. 193-225). New York, NY: Academic Press.

Hashtroudi, S., Johnson, M. K., \& Chrosniak, L. D. (1990). Aging and qualitative characteristics of memories for perceived and imagined complex events. Psychology and Aging, 5, 119-126. doi:10.1037/0882-7974 .5.1.119

Hay, J. F., \& Jacoby, L. L. (1999). Separating habit and recollection in young and older adults: Effects of elaborative processing and distinctiveness. Psychology and Aging, 14, 122-134. doi:10.1037/0882-7974 .14 .1 .122

Healy, M. R., Light, L. L., \& Chung, C. (2005). Dual-process models of associative recognition in young and older adults: Evidence from receiver operating characteristics. Journal of Experimental Psychology: Learning, Memory, and Cognition, 31, 768-788. doi:10.1037/02787393.31.4.768

Henkel, L. A., Johnson, M. K., \& De Leonardis, D. M. (1998). Aging and source monitoring: Cognitive processes and neuropsychological correlates. Journal of Experimental Psychology: General, 127, 251-268. doi:10.1037/0096-3445.127.3.251

Huynh, H., \& Feldt, L. S. (1976). Estimation of the box correction for degrees of freedom from sample data in randomized block and split-plot designs. Journal of Educational Statistics, 1, 69-82. doi:10.3102/ 10769986001001069

Jacoby, L. L., \& Rhodes, M. G. (2006). False remembering in the aged. Current Directions in Psychological Science, 15, 49-53. doi:10.1111/j .0963-7214.2006.00405.x

Johnson, M. K., Hashtroudi, S., \& Lindsay, D. S. (1993). Source monitoring. Psychological Bulletin, 114, 3-28.

Kausler, D. H. (1994). Learning and memory in normal aging. New York, NY: Academic Press.

Kausler, D. H., Salthouse, T. A., \& Saults, J. S. (1988). Temporal memory over the adult lifespan. The American Journal of Psychology, 101, 207-215.

Kelley, C. M., \& Sahakyan, L. (2003). Memory, monitoring, and control in the attainment of memory accuracy. Journal of Memory and Language, 48, 704-721. doi:10.1016/S0749-596X(02)00504-1

Kilb, A., \& Naveh-Benjamin, M. (2011). The effects of pure pair repetition on younger and older adults' associative memory. Journal of Experimental Psychology: Learning, Memory, and Cognition, 37, 706-719. doi:10.1037/a0022525

Koriat, A., \& Ackerman, R. (2010). Choice latency as a cue for children's subjective confidence in the correctness of their answers. Developmental Science, 13, 441-453. doi:10.1111/j.1467-7687.2009.00907.x

Koriat, A., \& Goldsmith, M. (1996). Monitoring and control processes in the strategic regulation of memory accuracy. Psychological Review, 103, 490-517. doi:10.1037/0033-295X.103.3.490
Koriat, A., Goldsmith, M., Schneider, W., \& Nakash-Dura, M. (2001). The credibility of children's testimony: Can children control the accuracy of their memory reports? Journal of Experimental Child Psychology, 79, 405-437. doi:10.1006/jecp.2000.2612

Kumaran, D., \& Maguire, E. A. (2009). Novelty signals: a window into hippocampal information processing. Trends in Cognitive Science, 13, 47-54. doi:10.1016/j.tics.2008.11.004

Lehrl, S. (1977). Mehrfachwahl-Wortschatz-Test B (MWT-B). Erlangen, Germany: Straube.

Lehrl, S. (2005). Mehrfachwahl-Wortschatz-Intelligenztest (MWT-B). Balingen, Germany: Spitta Verlag.

Li, S.-C., Lindenberger, U., Hommel, B., Aschersleben, G., Prinz, W., \& Baltes, P. B. (2004). Transformations in the couplings among intellectual abilities and constituent cognitive processes across the life span. Psychological Science, 15, 155-163. doi:10.1111/j.0956-7976.2004 .01503003.x

Light, L. L., Patterson, M. M., Chung, C., \& Healy, M. R. (2004). Effects of repetition and response deadline on associative recognition in young and older adults. Memory and Cognition, 32, 1182-1193.

Lindsay, D. S., \& Johnson, M. K. (1989). The eyewitness suggestibility effect and memory for source. Memory and Cognition, 17, 349-358.

Lindsay, D. S., Johnson, M. K., \& Kwon, P. (1991). Developmental changes in memory source monitoring. Journal of Experimental Child Psychology, 52, 297-318. doi:10.1016/0022-0965(91)90065-Z

Loftus, E. F. (2005). Planting misinformation in the human mind: a 30-year investigation of the malleability of memory. Learning and Memory, 12, 361-366. doi:10.1101/lm.94705

Loftus, E. F., Miller, D. G., \& Burns, H. J. (1978). Semantic integration of verbal information into a visual memory. Journal of Experimental Psychology: Human Learning and Memory, 4, 19-31.

May, C. P., Hasher, L., \& Kane, M. J. (1999). The role of interference in memory span. Memory \& Cognition, 27, 759-767.

Mitchell, K. J., \& Johnson, M. K. (2009). Source monitoring 15 years later: What have we learned from fMRI about the neural mechanisms of source memory? Psychological Bulletin, 135, 638-677. doi:10.1037/ a0015849

Mitchell, K. J., \& Zaragoza, M. S. (2001). Contextual overlap and eyewitness suggestibility. Memory \& Cognition, 29, 616-626.

Moscovitch, M., \& Winocur, G. (2002). The frontal cortex and working with memory. In D. T. Stuss \& R. T. Knight (Eds.), Principles of the frontal lobe function (pp. 188-209). New York, NY: Oxford University Press. doi:10.1093/acprof:oso/9780195134971.003.0012

Naveh-Benjamin, M. (1990). Coding of temporal order information: An automatic process? Journal of Experimental Psychology: Learning, Memory, and Cognition, 16, 117-126. doi:10.1037/0278-7393.16.1.117

Nelson, T. O., \& Narens, L. (1990). Metamemory: A theoretical framework and new findings. Psychology of Learning and Motivation, 26, 125-173. doi:10.1016/S0079-7421(08)60053-5

Ofen, N., Kao, Y. C., Sokol-Hessner, P., Kim, H., Whitfield-Gabrieli, S., \& Gabrieli, J. D. E. (2007). Development of the declarative memory system in the human brain. Nature Neuroscience, 10, 1198-1205. doi: $10.1038 / \mathrm{nn} 1950$

Paz-Alonso, P. M., Ghetti, S., Donohue, S. E., Goodman, G. S., \& Bunge, S. A. (2008). Neurodevelopmental correlates of true and false recognition. Cerebral Cortex, 18, 2208-2216. doi:10.1093/cercor/bhm246

Poole, D. A., \& Lindsay, D. S. (1995). Interviewing preschoolers: Effects of nonsuggestive techniques, parental couching and leading question on reports of nonexperienced events. Journal of Experimental Child Psychology, 60, 129-154. doi:10.1006/jecp.1995.1035

Rajah, M. N., \& McIntosh, A. R. (2008). Age-related differences in brain activity during verbal recency memory. Brain Research, 1199, 111-125. doi:0.1016/j.brainres.2007.12.051

Raz, N., Ghisletta, P., Rodrigue, K. M., Kennedy, K. M., \& Lindenberger, U. (2010). Trajectories of brain aging in middle-aged and older adults: 
Regional and individual differences. NeuroImage, 51, 501-511. doi: 10.1016/j.neuroimage.2010.03.020

Raz, N., Lindenberger, U., Rodrigue, K. M., Kennedy, K. M., Head, D., Williamson, A., .. A Acker, J. D. (2005). Regional brain changes in aging healthy adults: General trends, individual differences and modifiers. Cerebral Cortex, 15, 1676-1689. doi:10.1093/cercor/bhi044

Roebers, C. M. (2002). Confidence judgments in children's and adults' event recall and suggestibility. Developmental Psychology, 38, 10521067. doi:10.1037/0012-1649.38.6.1052

Roediger, H. L., III, \& Geraci, L. (2007). Aging and the misinformation effect: A neuropsychological analysis. Journal of Experimental Psychology: Learning, Memory, and Cognition, 33, 321-334. doi: 10.1037/0278-7393.33.2.321

Roediger, H. L., III, \& McDermott, K. B. (1995). Creating false memories: Remembering words not presented in lists. Journal of Experimental Psychology: Learning, Memory, and Cognition, 21, 803-814. doi: 10.1037/0278-7393.21.4.803

Rotello, C. M., \& Heit, E. (1999). Two-process models of recognition memory: Evidence for recall-to-reject? Journal of Memory \& Language, 40, 432-453.

Rybash, J. M., \& Hrubi-Bopp, K. L. (2000). Source monitoring and false recollection: A life span developmental perspective. Experimental Aging Research, 26, 75-87. doi:10.1080/036107300243696

Sander, M. C., Werkle-Bergner, M., \& Lindenberger, U. (2011). Binding and strategic selection in working memory: A lifespan dissociation. Psychology and Aging, 26, 612-624. doi:10.1037/a0023055

Schacter, D. L., Norman, K. A., \& Koutstaal, W. (1998). The cognitive neuroscience of constructive memory. Annual Review of Psychology, 49, 289-318. doi:10.1146/annurev.psych.49.1.289

Schneider, W. (1998). The development of procedural metamemory in childhood and adolescence. In G. Mazzoni \& T. O. Nelson (Eds.), Metacognition and cognitive neuropsychology: Monitoring and control processes (pp. 1-23). Mahwah, NJ: Erlbaum.

Schneider, W., \& Lockl, K. (2002). The development of metacognitive knowledge in children and adolescents. In T. Perfect \& B. Schwartz (Eds.), Applied metacognition (pp. 224-257). New York, NY: Cambridge University Press.

Schneider, W., \& Pressley, M. (1997). Memory development between two and twenty (2nd ed.). Mahwah, NJ: Erlbaum.

Schnider, A., von Daniken, C., \& Gutbrod, K. (1996). The mechanisms of spontaneous and provoked confabulations. Brain, 119, 1365-1375. doi: 10.1093/brain/119.4.1365

Shing, Y. L., \& Lindenberger, U. (2011). The development of episodic memory: Lifespan lessons. Child Development Perspectives, 5, $148-$ 155. doi:10.1111/j.1750-8606.2011.00170.x

Shing, Y. L., Werkle-Bergner, M., Brehmer, Y., Muller, V., Li, S. C., \& Lindenberger, U. (2010). Episodic memory across the lifespan: The contributions of associative and strategic components. Neuroscience and Biobehavioral Reviews, 34, 1080-1091. doi:10.1016/j.neubiorev.2009 .11 .002

Shing, Y. L., Werkle-Bergner, M., Li, S. C., \& Lindenberger, U. (2008). Associative and strategic components of episodic memory: A life-span dissociation. Journal of Experimental Psychology: General, 137, 495513. doi:10.1037/0096-3445.137.3.495

Shing, Y. L., Werkle-Bergner, M., Li, S. C., \& Lindenberger, U. (2009). Committing memory errors with high confidence: Older adults do but children don't. Memory, 17, 169-179. doi:10.1080/ 09658210802190596

Simons, J. S., \& Spiers, H. J. (2003). Prefrontal and medial temporal lobe interactions in long-term memory. Nature Reviews Neuroscience, 4, 637-648. doi:10.1038/nrn1178

Sluzenski, J., Newcombe, N. S., \& Kovacs, S. L. (2006). Binding, relational memory, and recall of naturalistic events: A developmental perspective. Journal of Experimental Psychology: Learning, Memory, and Cognition, 32, 89-100. doi:10.1037/0278-7393.32.1.89

Sluzenski, J., Newcombe, N., \& Ottinger, W. (2004). Changes in reality monitoring and episodic memory in early childhood. Developmental Science, 7, 225-245. doi:10.1111/j.1467-7687.2004.00341.x

Snodgrass, J. G., \& Corwin, J. (1988). Pragmatics of measuring recognition memory: Applications to dementia and amnesia. Journal of Experimental Psychology: General, 117, 34-50. doi:10.1037/0096-3445.117.1.34

Sowell, E. R., Peterson, B. S., Thompson, P. M., Welcome, S. E., Henkenius, A. L., \& Toga, A. W. (2003). Mapping cortical change across the human life span. Nature Neuroscience, 6, 309-315. doi:10.1038/nn1008

Sowell, E. R., Thompson, P. M., Leonard, C. M., Welcome, S. E., Kan, E., \& Toga, A. W. (2004). Longitudinal mapping of cortical thickness and brain growth in normal children. Journal of Neuroscience, 24, 82238231. doi:10.1523/JNEUROSCI.1798-04.2004

Spencer, W. D., \& Raz, N. (1995). Differential effects of aging on memory for content and context: A meta-analysis. Psychology and Aging, 10, 527-539. doi:10.1037/0882-7974.10.4.527

Tewes, U. (1991). Hamburg-Wechsler-Intelligenztest für Erwachsene (HAWIE-R). Bern, Switzerland: Hans Huber.

Wechsler, D. (1955). Wechsler adult intelligence scale manual. New York, NY: Psychological Corporation.

Wendelken, C., Baym, C. L., Gazzaley, A., \& Bunge, S. A. (2011). Neural indices of improved attentional modulation over middle childhood. Developmental Cognitive Neuroscience, 1, 175-186. doi:10.1016/j.den .2010.11.001

Received January 4, 2012

Revision received October 24, 2012

Accepted November 2, 2012 